\title{
Robust Regressive Feature Extraction Based Relevance Vector Margin Boosting For Aerial Images Scene Classification
}

\author{
K.Murugan
}

\begin{abstract}
Aerial image scene classification is a key problem to be resolved in image processing. Many research works have been designed for carried outing scene classification. But, accuracy of existing scene classification was lower. In order to overcome such limitation, a Robust Regressive Feature Extraction Based Relevance Vector Margin Boosting Scene Classification (RRFERVMBSC) Technique is proposed. The RRFE-RVMBSC technique is designed for improving the classification performance of aerial images with minimal time. The RRFERVMBSC technique comprises two main processes namely feature extraction and classification. Initially, RRFE-RVMBSC technique gets number of aerial images as input. After taking input, Robust Regressive Independent Component Analysis Based Feature Extraction process is performed in order to extract the features i.e. shape, color, texture and size from aerial image. After completing feature extraction process, RRFERVMBSC technique carried outs Ensembled Relevance Vector Margin Boosting Classification (ERVMBC) where all the input aerial images are classified into multiple classes with higher accuracy. The RRFE-RVMBSC technique constructs a strong classifier by reducing the training error of weak RVM classifier for effectual aerial images scene categorization. The RRFERVMBSC technique accomplishes simulation work using parameters such as feature extraction time classification accuracy and false positive rate with respect to number of aerial images.
\end{abstract}

Keywords: Aerial image, Relevance Vector Margin Boosting, Robust Regressive Independent Component Analysis, Strong classifier, Training Error, Weak RVM

\section{INTRODUCTION}

Scene classification attains greater significance in the remote sensing field. The aerial scene classification is difficult tasks in the remote sensing community. During classification process, each input aerial image is labeled into corresponding semantic. Recently, a lot of research works have been introduced for classifying aerial images. But, the classification performance of traditional works was not adequate. Besides to that, time taken for extracting relevant features using existing methods was more. In order to addresses the above drawbacks, RRFE-RVMBSC Technique is designed in this research work by using Robust Regressive Independent Component Analysis Based Feature Extraction (RRICA-FE) algorithm and Ensembled Relevance Vector Margin Boosting Classification (ERVMBC).

Revised Manuscript Received on April, 022020.

* Correspondence Author

Dr.K. Murugan*, Assistant Professor, Department of Computer Science, Government College for Women, Kolar, India.

(C) The Authors. Published by Blue Eyes Intelligence Engineering and Sciences Publication (BEIESP). This is an open access article under the CC BY-NC-ND license (http://creativecommons.org/licenses/by-nc-nd/4.0/)
Two-level feature representation (TLFR) model was presented in [1] for accomplishing aerial scene categorization. However, feature extraction time was more Global-local attention network (GLANet) was designed in [2] to extract global and local information for aerial scene classification. But, false positive rate was very higher.A Multitask Topological Codebook was utilized in [3] to enhance accuracy of large-scale aerial image classification with minimal time. However, classification performance was not sufficient. A novel method was introduced in [4] with aim of categorizing satellite images into multiple classes using support vector machine. But, misclassification error of this method was not reduced.

Two intensity order-based descriptors were applied in [5] to diminish the false positive rate of high-resolution aerial images categorization. However, ratio of number of aerial images correctly classified was minimal. Unsupervised Feature Learning was developed in [6] with the application to minimize computational complexity of aerial scene classification. But, classification accuracy of unsupervised feature learning was lower.

A convolutional neural network was presented in [7] to carry out high-resolution aerial image classification with minimal error. However, the processing time taken for image classification was not reduced. A contextual topological codebook was employed in [8] for assigning labels to aerial image. But, ratio of number of aerial images wrongly classified was lower.

A comparative analysis of diverse methods designed for performing aerial scene classification process was examined in [9]. A random forest classification was applied in [10] in order to minimize accuracy of aerial scene categorization. But, the classification time required for aerial image was more.

In order to addresses the above mentioned conventional issues, a novel technique called RRFE-RVMBSC Technique is proposed. The main contributions of RRFE-RVMBSC Technique is explained in below,

- To minimize time complexity during the processes of feature extraction process from input aerial image for effective aerial image scene classification as compared to existing works, Robust Regressive Independent Component Analysis Based Feature Extraction (RRICAFE) algorithm is proposed in RRFE-RVMBSC Technique. As, RRICA-FE algorithm is computationally efficient and needs less memory to efficiently mine key features from input aeries images on the contrary to existing works.

Published By:

Blue Eyes Intelligence Engineering \& Sciences Publication

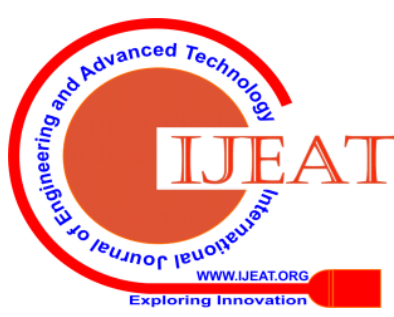


- To reduce the misclassification error of input aerial image scenes when compared to state-of-the-art works, Ensembled Relevance Vector Margin Boosting Classification (ERVMBC) algorithm is proposed in RRFERVMBSC Technique.

- The ERVMBC algorithm produce strong result with minimal training error for accurate classification of aerial image scenes.

The rest of the paper is created as follows; In Section 2, RRFE-RVMBSC Technique is explained with assist of architecture diagram. In Section 3, simulation settings are presented and the performance result of ADQBC-RHCDS Model is analysed in Section 4. Section 5 shows the literature survey. Section 6 shows the conclusion of the paper.

\section{LITERATURE SURVEY}

An automatic feature extraction was applied in [11] with help of an option to ground-based methods for aerial imagery. A recurrent neural network (RNN) classifier was employed in [12] to minimize the error of satellite image classification using the relevant features.

Block-based semantic classification method was designed in [13] for categorizing high quality multispectral aerial images with minimal computational time. A two-stream deep fusion system was developed in [14] with goal of classifying the aerial scene by application of ELM classifier.

An end-to-end trainable gated residual refinement network (GRRNet) was presented in [15] for removing irrelevant features and thereby increasing scene classification results. An unsupervised feature learning was applied in [16] with the intention of reducing false positive rate of aerial scene classification.

An enhanced cross-entropy method with an adaptive threshold was designed in [17] to decrease the training time complexity of aerial image categorization. A Deep Scene Representation was introduced in [18] to increase performance of aerial scene classification.

A survey of different deep networks developed for aerial scene classification was presented in [19]. Cross-domain distance metric learning (CDDML) framework was employed in [20] for scene categorization of aerial images.

\section{ROBUST REGRESSIVE FEATURE EXTRACTION BASED RELEVANCE VECTOR MARGIN BOOSTING SCENE CLASSIFICATION TECHNIQUE}

The Robust Regressive Feature Extraction Based Relevance Vector Margin Boosting Scene Classification (RRFERVMBSC) Technique is introduced in order to improve the aerial images scene classification performance with minimal false positive rate. On the contrary to traditional works, RRFE-RVMBSC Technique is developed by combining the Robust Regressive Independent Component Analysis Based Feature Extraction (RRICA-FE) algorithm and Ensembled Relevance Vector Margin Boosting Classification (ERVMBC). By using the RRICA-FE algorithm, RRFERVMBSC Technique reduces time complexity involved during the feature extraction process. In addition, with application of ERVMBC algorithm, RRFE-RVMBSC Technique minimizes training error of aerial images scene classification. The architecture diagram of RRFE-RVMBSC Technique is presented in Figure 1.

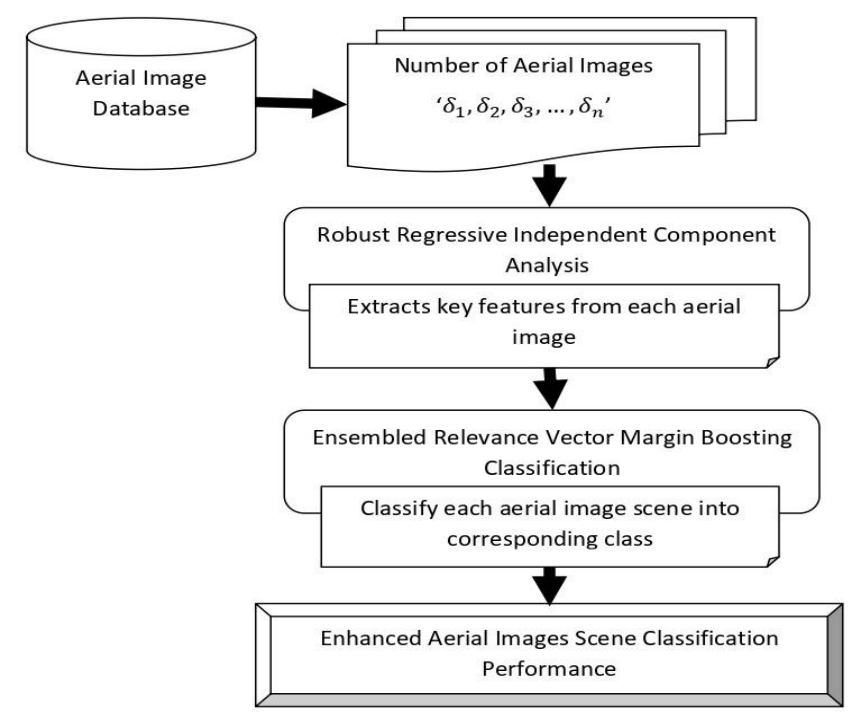

Figure 1 Architecture Diagram of RRFE-RVMBSC Technique for Aerial Images Scene Classification

Figure 1 shows the overall flow processes of RRFERVMBSC Technique. As depicted in Figure 1, RRFERVMBSC Technique at first takes aerial image database as input. This database contains many number of aerial image represented as ' $\delta_{1}, \delta_{2}, \delta_{3}, \ldots, \delta_{n}$ '. The RRFE-RVMBSC Technique mainly includes of two processes namely feature extraction and classification. During the feature extraction process, RRFE-RVMBSC Technique applies Robust Regressive Independent Component Analysis with aiming at extracting relevant features from input images with lower time utilization. During the classification process, RRFERVMBSC Technique applies RRFE-RVMBSC Technique applies with objective of classifying each aerial image scene into interrelated class with higher accuracy. From that, RRFE-RVMBSC Technique attains enhanced aerial images scene classification performance when compared to state-ofthe-art works. The detailed process of RRFE-RVMBSC Technique is described in below subsections.

\section{A. Robust Regressive Independent Component Analysis Based Feature Extraction}

The Robust Regressive Independent Component Analysis Based Feature Extraction (RRICA-FE) algorithm is designed in RRFE-RVMBSC Technique with the objective of increasing the feature extraction performance of input aerial images with a lower time complexity. On the contrary to state-of-the-art works, RRICA-FE algorithm is proposed by combining Robust Regression in independent component analysis. By using the robust regression concepts, RRICAFE algorithm significantly extract the features that are more useful to classify the scenes in input aerial images. Each input aerial image contains different features such as shape, texture, color, size, edges etc. The proposed RRICA-FE algorithm finds the features that are more significant to efficiently perform aerial images scene classification process with higher accuracy. 
The process involved in RRICA-FE algorithm is depicted in Figure 2.

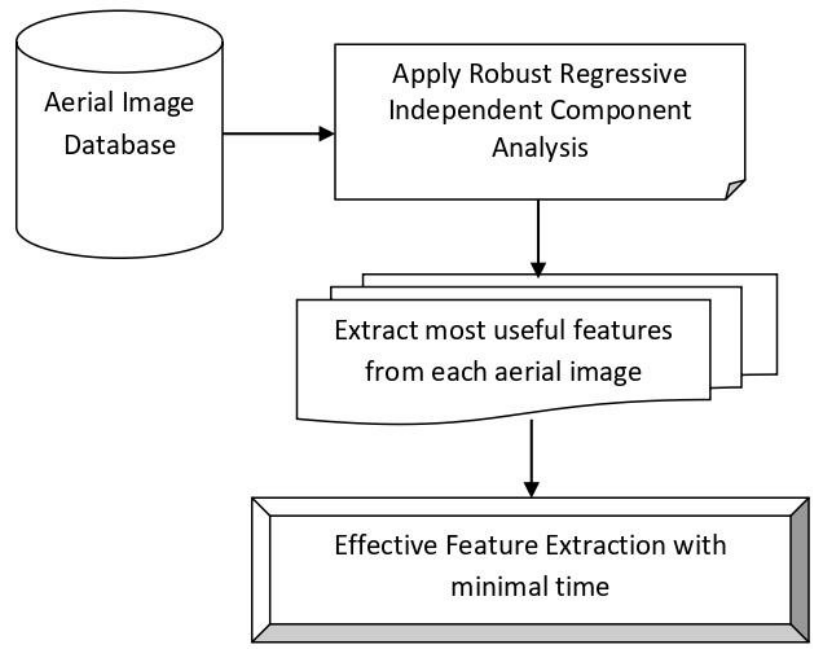

Figure 2 Robust Regressive Independent Component Analysis Process for Feature Extraction

Figure 2 shows the flow processes of RRICA-FE algorithm to get enhanced feature extraction performance. As demonstrated in the above figure, RRICA-FE algorithm at first takes number of aerial images as input. For each input aerial image ' $\delta_{i}$ ', then RRICA-FE algorithm apply independent component analysis in order to extract the object features with a lower amount time using below mathematical expression,

$$
z=w a
$$

From the above mathematical formula (1), ' $z$ ' represent an m-dimensional feature vector. Here, ' $a$ ' denotes the vector of n-dimensional independent components, ' $W$ ' indicates the weight matrix. In RRICA-FE algorithm, vector of ndimensional independent components is mathematically obtained using below,

$$
a=\varphi \cdot z
$$

From the above mathematical expression (2), ' $\varphi$ ' point outs a mapping function which helps for RRICA-FE algorithm to project the m-dimensional feature vector ' $z$ ' onto the subspace. Followed by, RRICA-FE algorithm utilized Gaussian kernel mapping function in a feature space of higher dimensions to efficiently extract objects features with help of below mathematical representation,

$$
\begin{gathered}
\varphi=\exp \left(-\alpha\left\|z-z^{y}\right\|^{2}\right) \\
\alpha=2 \sigma^{-2}
\end{gathered}
$$

From the above mathematical representations (3) and (4), ' $\sigma$ ' symbolizes variance, ' $z$ ' and ' $z$ ', refers the feature vectors in some input space ' $\left\|z-z^{\prime}\right\|^{2}$, denotes the squared Euclidean distance between the two feature vectors. By using the above mathematical equation, RRICA-FE algorithm projects the feature vector (i.e. object features) onto the subspace. Thus, proposed RRICA-FE algorithm accurately extracts feature that present in each aerial image for scene classification.

After discovering the objects features, RRICA-FE algorithm identifies relevant features by applying robust regression for effectual classification of aerial images. To significantly mine relevant features, the RRICA-FE algorithm determines the relationship between the extracted features with help of robust regression. Thus, robust regression is mathematically performed with help of below equation,

$$
z=b_{1}+b_{2} \cdot \delta_{i}
$$

From the above mathematical equation (5), ' $Z$ ' denotes labels to data (i.e. relevant or irrelevant feature) and ' $\hat{\delta}_{i}$ ' represents extracted features of aerial image. The RRICAFE discovers the best regression fit line via determining the best ' $b_{1}$ ' and ' $b_{2}$ ' values whereas ' $b_{1}$ ' signify intercept and ' $b_{2}$ ' designates coefficient of ' $\delta_{i}$ '. After identifying the best ' $b_{1}$ ' and ' $b_{2}$ ' values, RRICA-FE determines the best fit line. Based on this value, the RRICA-FE makes a decision to significantly extract the relevant features form each input aeries images with minimal time.

The algorithmic processes of RRICA-FE is explained in algorithm 1. Algorithm 1 shows the step by step processes of RRICA-FE algorithm. With the help of above algorithmic steps, RRFE-RVMBSC Technique increases the feature extraction performance in order to efficiently mine the more important features from each input aerial images with minimal amount of time utilization as compared to conventional works.

\section{// Robust Regressive Independent Component}

Analysis Based Feature Extraction Algorithm

Input: Number of Aerial Images ' $\delta_{1}, \delta_{2}, \delta_{3}, \ldots, \delta_{n}$ '

Output: Extract significant features with a lower amount of time

Step 1: Begin

Step 2: For each aerial image ' $\delta_{i}$ '

Step 3:Apply Independent Component Analysis using (1), (2), (3) and (4)

Step 4: Extract object features

Step 5: Apply robust regression using (5)

Step 6: If ' $(Z==1)$ ', then

Step 7: Feature is relevant for classification

Step 8: Else

Step 9: Feature is irrelevant

Step 10: End If

Step 11: Find outs most useful features for scene classification

\section{Step 12: End For}

Step 13:End

Algorithm 1 Robust Regressive Independent Component Analysis Based Feature Extraction

\section{B. Ensembled Relevance Vector Margin Boosting}

\section{Classification}

Ensembled Relevance Vector Margin Boosting Classification (ERVMBC) is designed in RRFE-RVMBSC Technique in order to reduce the false positive rate of aerial images scene classification. On the contrary to existing works, ERVMBC is proposed by applying margin boosting algorithm in relevance vector machine classification. The ERVMBC considers relevance vector machine (RVM) classification as weak classifier. The ERVMBC is a method of converting a set of weak RVM classifiers into strong classifier for efficient aerial image scene classification. The process involved in ERVMBC is depicted in below Figure 3. 


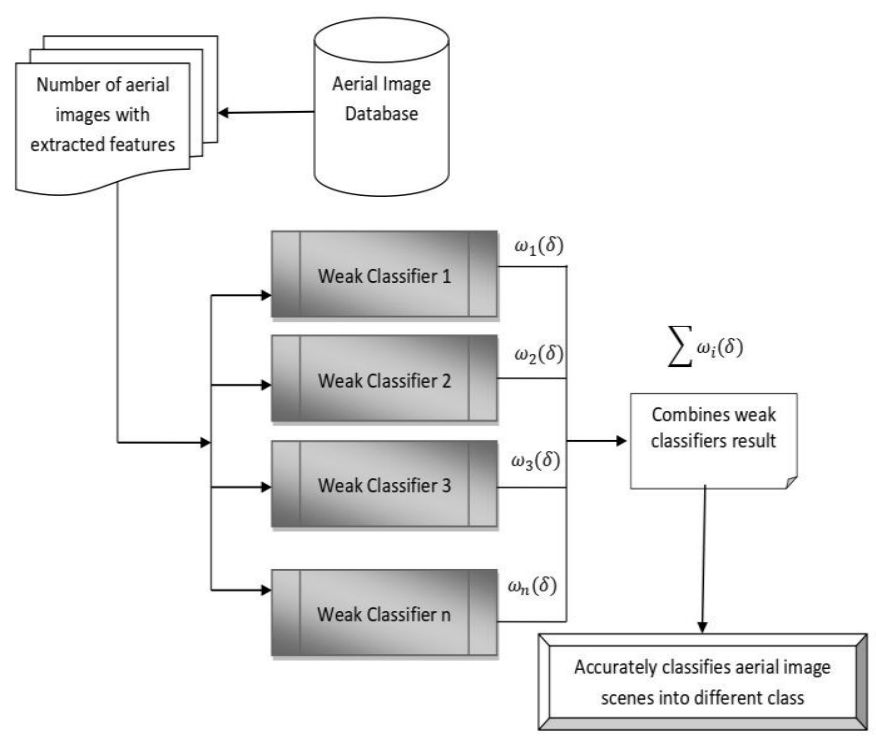

Figure 3 Flow processes of ERVMBC Algorithm

Figure 3 presents block diagram of ERVMBC algorithm for accurate scene classification. As demonstrated in above figure, ERVMBC initially get number of aerial images with extracted features as input. Then, ERVMBC generates ' $n$ ' weak RVM classification result for each input aerial image. In ERVMBC, each aerial image is trained using weak RVM to classify the scenes into diverse class. The weak RVM is represented as ' $\left\{\left(\delta_{1}, \omega_{1}\right),\left(\delta_{2}, \omega_{2}\right), \ldots .\left(\delta_{n}, \omega_{n}\right)\right\}$ ' where ' $\delta_{i}$ indicates an input aerial image and ' $\omega_{i}$ ' refers to the classification output. The RVM classification model is mathematically represented as follows,

$$
z\left(\delta_{i}, w\right)=\sum_{i=1}^{n} w_{i} K\left(\delta, \delta_{i}\right)+b_{0}
$$

From (6), ' $K\left(\delta, \delta_{i}\right)$ ' refers the kernel function and ' $w_{i}$ ' represents the weight of the ' $i^{t h}$, kernel function ' $w=\left[w_{0}, w_{1}, \ldots w_{n}\right]^{T}$. Here, ' $b_{0}$ ' indicates to the bias. In weak RVM classifier, Gaussian kernel function is utilized to map each input aerial image scene into a related class. In order to minimize the over-fitting due to excessive support vectors utilized in weak RVM classifier, all weight vectors must satisfy a zero-mean Gaussian prior distribution condition shown in below,

$$
\begin{aligned}
& P(w, v)=\prod_{i=0}^{n} n\left(w_{i} \mid 0, v_{i}^{-1}\right)= \\
& \prod_{i=0}^{n} \frac{\vartheta_{i}}{\sqrt{2 \pi}} \exp \left(-\frac{v_{i} w_{i}^{3}}{2}\right)
\end{aligned}
$$

From (7), ' $v=\left[v_{0}, v_{1}, v_{2}, v_{n}\right]^{T}$, denotes the hyperparameter vector that determines the prior distribution of weight vector ' $w$ ' and controls the degree to which the weight diverges from its zero-mean for classify each input image into a multiple classes. By using the equation (6) and (7), weak RVM classifier categorizes all the input aerial image scenes into different class. But, false positive rate of weak RVM classifier is higher. To enhances the classification performance, then weak RVM classifier results are aggregated into one strong classifier using below mathematical formula,

$$
S(\delta)=\sum_{i=1}^{n} \omega_{i}(\delta)
$$

From the above mathematical representation (8), ' $S(\delta)$, indicates the results of the strong classifier in which
' $\omega_{i}(\delta)$ ' refers to an output of weak RVM classifier. After uniting the weak RVM classification result, the same weight is initialized in ERVMBC for all weak RVM classifier using below mathematical expression.

$$
S(\delta)=\sum_{i=1}^{n} \varepsilon * \omega_{i}(\delta)
$$

From the above mathematical equation (9), ' $\varepsilon_{i}$ ' denotes the initial weight of weak RVM classifier ' $\omega_{i}(\delta)$ '. Subsequently, ERVMBC evaluates the training error for each weak RVM classifier as difference between the actual and predicted result using below mathematical representation.

$$
\tau_{e}=\left(\omega_{0}(\delta)-\omega_{i}(\delta)\right)^{2}
$$

From the above mathematical expression (10), ' $\tau_{e}$ ' signifies a measured training error of the weak RVM classifier. Here, ' $\omega_{0}(\delta)$ ' refers to the actual result of the weak RVM classifier and ' $\omega_{i}(\delta)$ ' point outs a predicted result of weak RVM classifier. According to the estimated error value, then weight of each weak RVM classifier is updated. The initial weight gets maximized when the weak RVM classifier wrongly classified the input aerial image scenes. Likewise, the initial weight gets reduced when the weak classifier exactly classified the input aerial image scenes.

After updating the weight value, the ERVMBC finds the weak RVM classifier with minimal training error as strong classifier by applying gradient descent function using below mathematical expression,

$$
F=\operatorname{argmin} \tau_{e}\left(\omega_{i}(\delta)\right)
$$

From the above mathematical formula (11), ' $F$ ' denotes the gradient descent function. Here, ' $\arg \min$ ' represents the argument of the minimum which assists to discover weak RVM classifier ' $\omega_{i}(\delta)$ ' with lower training error. The final strong classifier result is obtained through weighted linear combination of the results of the weak RVM classifier using below,

$$
S(\delta)=\sum_{i=1}^{n} \varepsilon^{\prime \prime} * \omega_{i}(\delta)
$$

From the above mathematical expression (12), ' $\varepsilon$ ', symbolize the updated weight of the weak classifier ' $\omega_{i}(\delta)$ '. From the above equation, ERVMBC exactly classifies each input aerial images into corresponding class with higher accuracy and minimal time.

The algorithmic process of ERVMBC is described below,

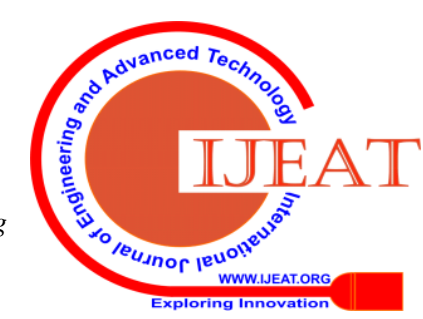


// Ensembled Relevance Vector Margin Boosting Classification

Input: Number of Aerial Images ' $\delta_{1}, \delta_{2}, \delta_{3}, \ldots, \delta_{n}$ '

Output: achieve higher scene classification accuracy

Step 1: Begin

Step 2: For each input aerial image ' $\delta$ ' with extracted relevant features

Step 3: Get ' $n$ ' number of weak RVM classifiers result

Step 4: For each weak RVM classifier ' $\omega_{i}(\delta)$ '

Step 5: Initialize the weight ' $\varepsilon$ '

Step 6: Determine training error ' $\tau_{e}$ '

Step 7: Update the weight ' $\varepsilon^{n}$,

Step 8: End For

Step 9: Identify weak RVM classifier with minimum error ' $\arg \min \tau_{\varepsilon}\left(\omega_{i}(\delta)\right)$ '

Step 10: Obtains strong classification

results ' $S(\delta)=\sum_{i=1}^{n} \varepsilon * \omega_{i}(\delta)$ '

Step 11: Accurately classifies aerial image scenes into a corresponding class

Step 12: End For

Step 13:End For

Algorithm 2 Ensembled Relevance Vector Margin Boosting Classification

Algorithm 2 shows step by step processes of ERVMBC to achieve better aerial image scene classification performance. By using the above algorithmic process of ERVMBC, proposed RRFE-RVMBSC technique generates strong classification result in order to effectually classify all the input aerial images into a multiple class with a minimal error. This supports for proposed RRFE-RVMBSC technique to get enhanced classification performance with a minimal amount of time consumption for aerial images when compared to conventional works.

\section{SIMULATION SETTINGS}

To determine the performance, proposed RRFE-RVMBSC technique and conventional Two-level feature representation (TLFR) [1] and global-local attention network (GLANet) [2] are implemented in MATLAB simulator by using Aerial Image database. This database is taken from http://dsp.etfbl.net/aerial/ which includes 606 RGB aerial images with size of $128 * 128$ pixels. Besides, Aerial Image database comprises of buildings, factories, and warehouses, as well as natural, such as fields, trees and rivers. In order to conduct simulation work, RRFERVMBSC technique considers different number of aerial images in the range of 25-250 from this database. The efficiency of RRFE-RVMBSC technique is estimated in terms of feature extraction time, scene classification accuracy and false positive rate with respect o various number of input images. The simulation result of RRFERVMBSC technique is compared against with two traditional works namely Two-level feature representation (TLFR) [1] and global-local attention network (GLANet) [2].

\section{RESULT ANALYSIS}

In this section, the simulation result of RRFE-RVMBSC technique is presented. The performance result of RRFERVMBSC technique is compared with Two-level feature representation (TLFR) [1] and global-local attention network (GLANet) [2] respectively using below parameters with the help of tables and graphical representation.

\section{A. Case 1: Feature Extraction Time}

In proposed RRFE-RVMBSC technique, Feature extraction time (FET) determine the amount of time utilized to extract the shape, color, texture, size of object features from the input aerial images. The feature extraction time is mathematically calculated using below,

$$
F E T=n \text { time }(E S)
$$

From the above formula (13), 'time(ES)' denotes an amount of time consumed for extracting features from a single aerial image and ' $n$ ' denotes a total number of images. The feature extraction time is evaluated in terms of milliseconds (ms).

\section{Sample Mathematical Calculation for Feature Extraction Time:}

- Proposed RRFE-RVMBSC: total number of aerial images are 25 and the time needed to find the features from single image is $1.05 \mathrm{~ms}$, then feature extraction time is measured as,

$$
F E T=25 * 1.05 \mathrm{~ms}=26 \mathrm{~ms}
$$

- Existing TLFR: total number of aerial images are 25 and the time required to discover the features from single image is $1.3 \mathrm{~ms}$, then feature extraction time is computed as,

$$
F E T=25: 1.3 \mathrm{~ms}=33 \mathrm{~ms}
$$

- Existing GLANet: total number of aerial images are 25 and the time utilized to identify the features from single image is $1.5 \mathrm{~ms}$, then feature extraction time is obtained as,

$$
F E T=25 * 1.5 \mathrm{~ms}=38 \mathrm{~ms}
$$

The performance result of feature extract time during the processes of aerial image scene classification process using three methods namely proposed RRFE-RVMBSC technique and conventional TLFR [1] and GLANet [2] is presented in below Table 1.

\section{Table 1 Tabulation for Feature Extraction Time}

\begin{tabular}{|c|c|c|c|}
\hline \multirow{2}{*}{$\begin{array}{c}\text { Number of } \\
\text { aerial images } \\
(\mathrm{n})\end{array}$} & \multicolumn{3}{|c|}{ Feature Extraction Time (ms) } \\
\cline { 2 - 4 } & $\begin{array}{c}\text { RRFE- } \\
\text { RVMBSC }\end{array}$ & TLFR & GLANet \\
\hline 25 & 14 & 16 & 21 \\
\hline 50 & 21 & 24 & 27 \\
\hline 75 & 26 & 31 & 34 \\
\hline 100 & 31 & 38 & 41 \\
\hline 125 & 35 & 40 & 45 \\
\hline 150 & 38 & 44 & 47 \\
\hline 175 & 40 & 46 & 49 \\
\hline 200 & 44 & 48 & 52 \\
\hline 225 & 47 & 52 & 56 \\
\hline 250 & 50 & 55 & 60 \\
\hline
\end{tabular}

Figure 4 shows graphical result analysis of feature extraction time for effective aerial image scene classification with respect to various numbers of aerial images in the range of 25-250 using three methods i.e. proposed RRFERVMBSC technique and existing TLFR [1] and GLANet [2].

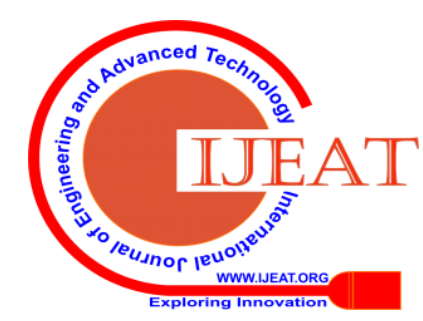


As demonstrated in the above figure, proposed RRFERVMBSC technique achieves better feature feature time with increasing number of aerial images as input for exactly classifying aerial images scenes when compared to TLFR [1] and GLANet [2]. This is owing to application of Robust Regressive Independent Component Analysis Based Feature Extraction (RRICA-FE) algorithm in proposed RRFERVMBSC technique on the contrary to state-of-the-art works.

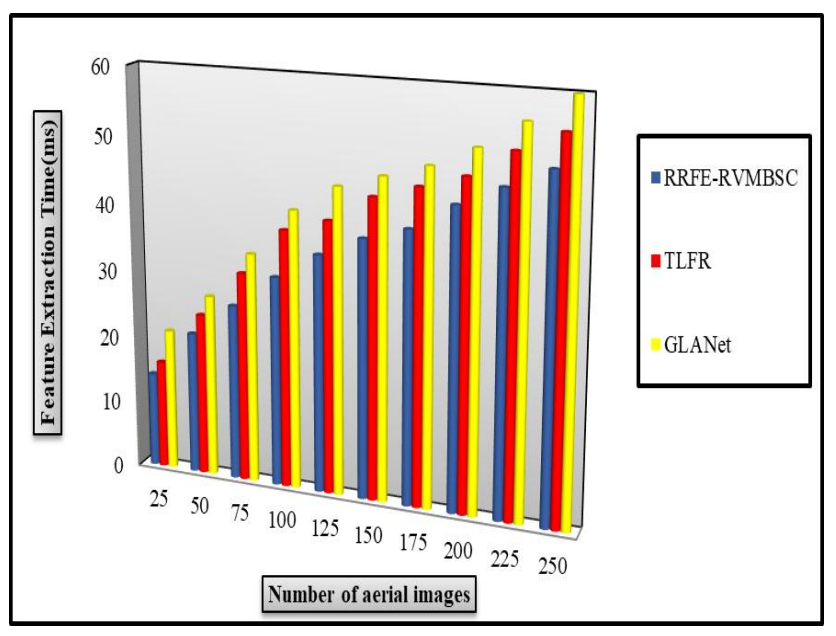

Figure 4 Experimental Result of Feature Extraction Time versus Number of aerial images

With the algorithmic concepts of RRICA-FE, proposed RRFE-RVMBSC technique discovers the features that are more useful to efficiently accomplish aerial images scene classification process with higher accuracy using robust regression. From that, proposed RRFE-RVMBSC technique minimize amount of time needed to extract the shape, color, texture, size of object features from the input aerial images when compared to other TLFR [1] and GLANet [2]. Hence, proposed RRFE-RVMBSC technique minimizes feature extraction time of aerial image scene classification by $13 \%$ and $21 \%$ when compared to TLFR [1] and GLANet [2] respectively.

\section{B. Case 2: Scene Classification Accuracy}

In proposed RRFE-RVMBSC technique, Scene Classification accuracy ( $S C A$ ) determines the ratio of number of aerial images scenes precisely classified to the total number of images. The scene classification accuracy is mathematically measured using below:

$$
S C A=\frac{n_{E C}}{n} * 100
$$

From the above equation (14), ' $n_{E C}$ ' point outs number of aerial images scenes exactly classified and ' $n$ ' refers total number of images. The scene classification accuracy is determined in terms of percentage (\%).

\section{Sample Calculation for Scene Classification Accuracy:}

- Proposed RRFE-RVMBSC: Number of aerial images scenes correctly classified is 23 and the total number of the images is 25, then the scene classification accuracy is obtained as,

$$
\text { SCA }=\frac{23}{25} \cdot 100=92 \%
$$

- Existing TLFR: Number of aerial images scenes accurately classified is 18 and the total number of the images is 25, then the scene classification accuracy is evaluated as,

$$
S C A=\frac{18}{25}: 100=72 \%
$$

- Existing GLANet: Number of aerial images scenes rightly classified is 21 and the total number of images is 25 , then the scene classification accuracy is computed as,

$$
S C A=\frac{21}{25} * 100=84 \%
$$

The simulation result of aerial image scene classification process using three methods namely proposed RRFERVMBSC technique and existing TLFR [1] and GLANet [2] is demonstrated in below Table 2.

Table 2 Tabulation for Scene Classification Accuracy

\begin{tabular}{|c|c|c|c|}
\hline \multirow{2}{*}{$\begin{array}{c}\text { Number of } \\
\text { aerial images (n) }\end{array}$} & \multicolumn{3}{|c|}{ Scene Classification Accuracy (\%) } \\
\cline { 2 - 4 } & $\begin{array}{c}\text { RRFE- } \\
\text { RVMBSC }\end{array}$ & TLFR & GLANet \\
\hline 25 & 92 & 72 & 84 \\
\hline 50 & 94 & 80 & 84 \\
\hline 75 & 96 & 85 & 87 \\
\hline 100 & 94 & 85 & 88 \\
\hline 125 & 95 & 87 & 90 \\
\hline 150 & 94 & 85 & 89 \\
\hline 175 & 95 & 89 & 91 \\
\hline 200 & 95 & 88 & 90 \\
\hline 225 & 96 & 86 & 88 \\
\hline 250 & 97 & 89 & 90 \\
\hline
\end{tabular}

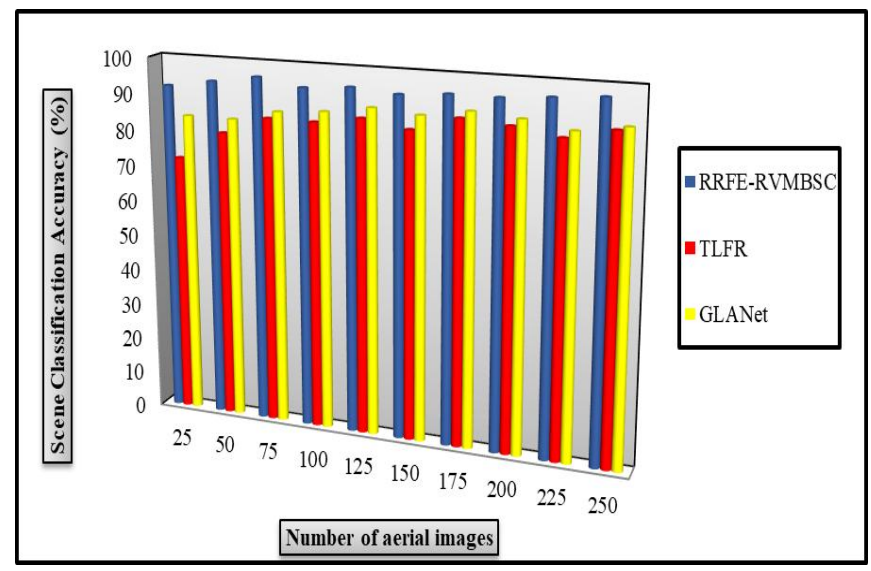

Figure 5 Experimental Result of Scene Classification Accuracy versus Number of aerial images

Figure 5 illustrates graphical result analysis of aerial image scene classification accuracy based on diverse numbers of aerial images in the range of 25-250 using three methods i.e. proposed RRFE-RVMBSC technique and existing TLFR [1] and GLANet [2]. As presented in the above figure, proposed RRFE-RVMBSC technique attains better scene classification accuracy with increasing number of aerial images as input for precisely categorizing aerial images scenes into a associtaed class when compared to TLFR [1] and GLANet [2]. This is because of application of Ensembled Relevance Vector Margin Boosting Classification (ERVMBC) algorithm in proposed RRFERVMBSC technique on the contrary to conventional works. 
By using algorithmic steps of ERVMBC, proposed RRFERVMBSC technique gets strong scene classification result by combining number of weak RVM classifer. The discover strong classifier contains very low training error as compared to other existing works [1] and [2]. Therefore, proposed RRFE-RVMBSC technique gives better aerial image scene classification result with higher accuracy. Thus, proposed RRFE-RVMBSC technique enhances the ratio of number of aerial images scenes precisely classified when compared to other TLFR [1] and GLANet [2]. As a result, proposed RRFE-RVMBSC technique increases scene classification accuracy by $12 \%$ and $8 \%$ when compared to TLFR [1] and GLANet [2] respectively.

\section{Case 3: False Positive Rate}

In proposed RRFE-RVMBSC technique, false positive rate (FPR) computes the ratio of a number of aerial images scenes wrongly classified to the total number of images. The false positive rate of aerial image scene classification is mathematically estimated as,

$$
F P R=\frac{n_{M C}}{n} ; 100
$$

From the above expression (15), ' $n_{M C}$ ' signifies number of aerial images scenes mistakenly classified and ' $n$ ' denotes total number of images. The false positive rate is estimated in terms of percentage (\%).

\section{Sample Mathematical Calculation for False Positive Rate:}

- Proposed RRFE-RVMBSC: Number of aerial images scenes erroneously classified is 2 and the total number of the images is 25 , then the false positive rate is calculated as,

$$
\text { FPR }=\frac{2}{25} * 100=8 \%
$$

- Existing TLFR: Number of aerial images scenes imperfectly classified is 7 and the total number of the images is 25, then the false positive rate is determined as,

$$
F P R=\frac{7}{25} * 100=28 \%
$$

- Existing GLANet: Number of aerial images scenes imprecisely classified is 4 and the total number of images is 25 , then the false positive rate is evaluated as,

$$
\mathrm{FPR}=\frac{4}{25} * 100=16 \%
$$

The comparative result of false positive rate for aerial image scene categorization using three methods namely proposed RRFE-RVMBSC technique and traditional TLFR [1] and GLANet [2] is depicted in below Table 3.

Table 3 Tabulation for False Positive Rate

\begin{tabular}{|c|c|c|c|}
\hline \multirow{2}{*}{$\begin{array}{c}\text { Number of } \\
\text { aerial images } \\
(\mathrm{n})\end{array}$} & \multicolumn{3}{|c|}{ False Positive Rate (\%) } \\
\cline { 2 - 4 } & $\begin{array}{c}\text { RRFE- } \\
\text { RVMBSC }\end{array}$ & TLFR & GLANet \\
\hline 25 & 8 & 28 & 16 \\
\hline 50 & 6 & 20 & 16 \\
\hline 75 & 4 & 15 & 13 \\
\hline 100 & 6 & 15 & 12 \\
\hline 125 & 5 & 13 & 10 \\
\hline 150 & 6 & 15 & 11 \\
\hline 175 & 5 & 11 & 9 \\
\hline 200 & 6 & 13 & 11 \\
\hline
\end{tabular}

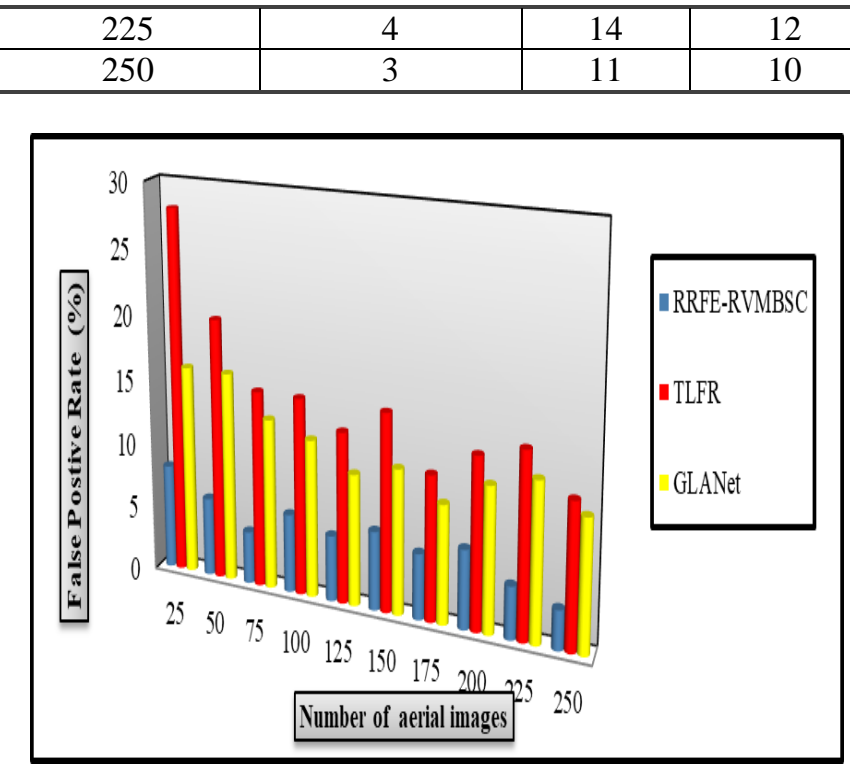

Figure 6 Experimental Result of False Positive Rate versus Number of aerial images

Figure 6 presents graphical result analysis of false positive rate for aerial image scene categorization along with varied numbers of aerial images in the range of 25-250 using three methods i.e. proposed RRFE-RVMBSC technique and traditonal TLFR [1] and GLANet [2]. As depicted in the above figure, proposed RRFE-RVMBSC technique obtains lower false posive rate with increasing number of aerial images as input for perfectly classify all the input aerial images scenes into a interrelated class when compared to TLFR [1] and GLANet [2]. This is due to application of Ensembled Relevance Vector Margin Boosting Classification (ERVMBC) algorithm in proposed RRFERVMBSC technique on the contrary to existing works.

With help of ERVMBC algorithm, proposed RRFERVMBSC technique measure training error for each weak RVM classifier and consequnenly updates weight of weak RVM classifier. In proposed RRFE-RVMBSC technique, initial weight gets increased when the weak RVM classifier mistakenly classified the input aerial image scenes. Similarly, the initial weight gets decreased when the weak classifier correctly classified the input aerial image scenes. After updating the weight value, the ERVMBC finds strong classifier through weighted linear combination of the results of the weak RVM classifier. From that, RRFE-RVMBSC technique obtains enhanced scene classification accuracy. Hence, proposed RRFE-RVMBSC technique minimizes the ratio of number of aerial images scenes wrongly classified when compared to other TLFR [1] and GLANet [2]. Accordingly, proposed RRFE-RVMBSC technique reduces false positve rate of scene classification by $65 \%$ and $55 \%$ when compared to TLFR [1] and GLANet [2] respectively.

\section{CONCLUSION}

The RRFE-RVMBSC Technique is developed with the intention of enhancing scene classification accuracy of aerial image with lower feature extraction. 
The aim of RRFE-RVMBSC Technique is attained with the application of Robust Regressive Independent Component Analysis Based Feature Extraction (RRICA-FE) algorithm and Ensembled Relevance Vector Margin Boosting Classification (ERVMBC). The proposed RRFE-RVMBSC Technique lessens amount of time taken to find out the shape, color, texture, size of object features from the input aerial images when compared to conventional work by using RRICA-FE algorithm. Also, proposed RRFE-RVMBSC Technique improves the ratio of number of aerial images scenes perfectly classified when compared to existing work with the application of ERVMBC algorithm. Moreover, proposed RRFE-RVMBSC Technique decreases the ratio of number of aerial images scenes incorrectly classified when compared to state-of-the-art work through using RRICA-FE and ERVMBC algorithm concepts. The simulation results depict that the proposed RRFE-RVMBSC Technique gives better performance with an enhanced scene classification accuracy and minimization of feature extraction time when compared conventional works.

\section{REFERENCES}

1. Jinrui Gan, Qingyong Li, Zhen Zhang, Jianzhu Wang, "Two-Level Feature Representation for Aerial Scene Classification", IEEE Geoscience and Remote Sensing Letters, Volume 13, Issue 11, Pages 1626 - 1630, 2016.

2. Yiyou Guo, Jinsheng Ji, Xiankai Lu, Hong Huo, Tao Fang, Deren Li, "Global-Local Attention Network for Aerial Scene Classification", IEEE Access, Volume 7, Pages 67200 - 67212, May 2019.

3. Luming Zhang, Meng Wang, Richang Hong, Bao-Cai Yin, Xuelong Li, "Large-Scale Aerial Image Categorization Using a Multitask Topological Codebook", IEEE Transactions on Cybernetics, Volume, 46, Issue, 2, Pages 535 - 545, 2016.

4. Giriraja C.V, Srinivasa C, T.K. Jaya Ram, Avula Haswanth, "Unsupervised Learning for Satellite Image Classification", IOSR Journal of VLSI and Signal Processing (IOSR-JVSP), Volume 4, Issue 2, Pages 01-04, 2014.

5. Wei Huang, Lingd, Wub Yingmei Wei, Hanchen Song, "Order based feature description for high-resolution aerial image classification", Optik - International Journal for Light and Electron Optics, Elsevier, Volume 125, Issue 24, Pages 7239-7243, December 2014.

6. Anil M. Cheriyadat, "Unsupervised Feature Learning for Aerial Scene Classification", IEEE Transactions on Geoscience and Remote Sensing, Volume 52, Issue 1, Pages 439 - 451, 2014.

7. Emmanuel Maggiori, Yuliya Tarabalka, Guillaume Charpiat, Pierre Alliez, "High-Resolution Aerial Image Labeling with Convolutional Neural Networks", IEEE Transactions on Geoscience and Remote Sensing, Volume 55, Issue 12, Pages 7092 -7103, 2017.

8. Zhijun Meng, Yan Wang, Xinyu Wu, Yating Yin, Teng Li, "Contextual aerial image categorization using codebook", Journal of Visual Communication and Image Representation, Elsevier, Volume 48, Pages 404-410, October 2017.

9. Gui-Song Xia, Jingwen Hu, Fan Hu, Baoguang Shi, Xiang Bai, Yanfei Zhong, Liangpei Zhang and Xiaoqiang Lu, "AID: A Benchmark Data Set for Performance Evaluation of Aerial Scene Classification", IEEE Transactions on Geoscience and Remote Sensing, Volume 55, Issue 7, Pages 3965 - 3981, July 2017.

10. Long Li, Carmen Solana, Frank Canters, Matthieu Kervyn, "Mapping lava flows from satellite remote sensing imagery through random forest classification", Journal of Volcanology and Geothermal Research, Elsevier, Pages 1-39, 2017.

11. Nathaniel Williams, Duncan Quincey and John Stillwell, "Automatic Classification of Roof Objects from Aerial Imagery of Informal Settlements in Johannesburg", Applied Spatial Analysis and Policy, Springer, Volume 9, Issue 2, Pages 269-281, June 2016.

12. Emmanuel Maggiori, Guillaume Charpiat, Yuliya Tarabalka, and Pierre Alliez, "Recurrent Neural Networks to Correct Satellite Image Classification Maps", IEEE Transactions on Geoscience and Remote Sensing, Volume 55, Issue 10, Pages 5913 - 5924, October 2017.

13. Aleksej Avramovic and Vladimir Risojevic, "Block-based semantic classification of high-resolution multispectral aerial images", Signal, Image and Video Processing, Springer, Volume 10, Issue 1, Pages 75-84, January 2016.
14. Yunlong $\mathrm{Yu}$ and Fuxian Liu, "A Two-Stream Deep Fusion Framework for High-Resolution Aerial Scene Classification", Computational Intelligence and Neuroscience, Hindawi, Volume 2018, Pages 1-13, January 2018.

15. JianfengHuang, XinchangZhang, QinchuanXin, YingSun, PengchengZhang, "Automatic Building Extraction from HighResolution Aerial Images and LiDARData using Gated Residual Refinement Network", ISPRS Journal of Photogrammetry and Remote Sensing, Elsevier, Volume 151, Pages 91-105, May 2019.

16. Anil M. Cheriyadat, "Unsupervised Feature Learning for Aerial Scene Classification", IEEE Transactions on Geoscience and Remote Sensing, Volume, 52, Issue 1, Pages 439 - 451, 2014.

17. Chu He, Bokun He, Xiaohuo Yin, Wenwei Wang, Mingsheng Liao, "Relationship Prior and Adaptive Knowledge Mimic Based Compressed Deep Network for Aerial Scene Classification", IEEE Access, Volume 7, Pages 137080 - 137089, July 2019.

18. Xiangtao Zheng, Yuan Yuan, Xiaoqiang Lu, "A Deep Scene Representation for Aerial Scene Classification", IEEE Transactions on Geoscience and Remote Sensing, Volume 57 , Issue 7, Pages 4799 - 4809, July 2019.

19. Muhammet Ali Dede, Erchan Aptoula, Yakup Genc, "Deep Network Ensembles for Aerial Scene Classification”, IEEE Geoscience and Remote Sensing Letters, Volume 16, Issue 5, Pages 732 - 735, May 2019.

20. Li Yan, Ruixi Zhu, Nan Mo, Yi Liu, "Cross-Domain Distance Metric Learning Framework with Limited Target Samples for Scene Classification of Aerial Images", IEEE Transactions on Geoscience and Remote Sensing, Volume 57, Issue 6, Pages 3840 - 3857, June 2019.

\section{AUTHOR PROFILE}

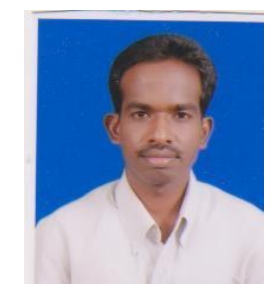

Dr.K. Murugan is currently working as Assistant Professor, Department of Computer Science, Government College for Women, Kolar, India. He has a teaching experience of 21 years. His area of interest includes Computer Networks, Image Processing, Mobile Computing and Machine Learning.

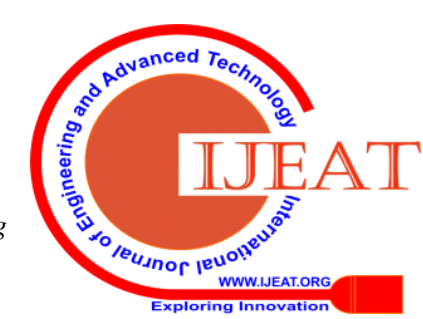

